\title{
GÉNERO, VEJEZ Y SALUD
}

\section{Melba Barrantes Monge*}

Resumen: Es necesario considerar los principios de beneficencia y justicia para una atención individual de las mujeres mayores y la formación de sistemas sanitarios (o políticas sanitarias) de carácter ético donde prevalezca siempre la equidad. Para ser justo y beneficente es imprescindible ser eficiente, y ello se alcanza tomando en cuenta factores propios de las mujeres ancianas: salud física y mental, estado de vida, real acceso a los servicios, entre otros.

Palabras clave: envejecimiento, género, salud, justicia y beneficencia

\section{GENDER, ELDERLY AND HEALTH CARE}

\begin{abstract}
It is necessary to consider the bioethical principles of beneficence and justice for giving individual health care to elderly women and for generating a health care system (or public policies) with an ethical approach where equity will be always present. In order to be fair and beneficent one must be efficient, which is achieved considering particular factors of the elderly: physical and mental health, quality of life, their real access to the health care services among others.
\end{abstract}

Key words: elderly, gender, health, justice and beneficence

\section{GÊNERO, VELHICE E SAÚDE}

Resumo: É necessário considerar os princípios de beneficência e justiça para uma atenção individual das mulheres idosas e a formação de sistemas de saúde (ou políticas sanitarias) de caráter ético, em que prevaleça a equidade. Para ser justo e beneficente é imprescindível ser eficiente e isto se alcança levando-se em conta os fatores próprios das mulheres idosas: saúde física e mental, estado de vida, acesso aos serviços, entre outros fatores

Palavras chave: enfelhecimento, gênero, saúde, justiça e beneficencia 


\section{Introducción}

Para evaluar la salud durante el envejecimiento es necesario tomar en cuenta el género, porque ello nos permite reconocer las dificultades y desigualdades en este grupo. En una sociedad donde no existe cultura de envejecimiento (como es el caso de la mayoría de los países latinoamericanos) y en la cual, a través de la historia, ser mujer ha constituido una desventaja social, resulta necesario enfocar éticamente la atención individual de este grupo de la población y formular políticas de salud atingentes. No obstante, esto no ocurre en la mayoría de los casos: se genera atención o políticas de salud para mujeres sin hacer separación por edad, o para el adulto mayor sin discriminar por género. Por lo tanto, las adultas mayores no reciben la atención adecuada o, en el peor de los casos, quedan excluidas de ella.

\section{Género y vejez}

Existen desigualdades en relación con el género y con la edad. Estas últimas deben tratarse desde el punto de vista bioético dentro del acápite de equidad y justicia. Son problemas que, usualmente, se abordan por separado; tal desconexión dificulta el diagnóstico, intervención, tratamiento y soluciones de las consecuencias de este proceso en las funciones, relaciones e identidades de ancianas y ancianos.

¿Por qué la edad, el género y la etnia se encuentran excluidos del discurso científico? Esto forma parte de una perspectiva masculina y los resultados se utilizan posteriormente (en forma errónea) como válidos para la generalidad de los individuos, hombres y mujeres, de cualquier condición (joven o viejo).

Generalmente se percibe el envejecimiento como deterioro mental y físico, consideración que es común tanto entre profesionales de salud como entre el público en general. Pero, en realidad, el envejecimiento es un proceso complejo: una persona es considerada "mayor" cuando alcanza la edad de 60-65 años, independientemente de su historia clínica y situación particular.

En el plano mundial la población está envejeciendo. La mayoría de esa población son mujeres, especialmente en el grupo mayor de 80 años. Si bien es cierto la mujer vive más, esto no indica que viva mejor, sino todo lo contrario. Los problemas surgidos a lo largo de la historia personal, como: mala nutrición, embarazos repetidos, eventuales abortos inseguros, falta de atención de algunas enfermedades médicas, violencia, problemas psicológicos no tratados, trabajos de múltiples jornadas, entre otros, cobran un alto precio durante esta etapa de la vida. Esa cantidad de años se acompaña de más morbilidades $\mathrm{y}$, sin embargo, tienen menos acceso a atención en salud. Esto se debe a los ingresos precarios de las ancianas, porque no cuentan con previsión social o porque, en muchas ocasiones, sus trabajos no han sido remunerados.

La variable género es crucial para estudiar enfermedad, salud y calidad de vida. No son iguales los desafíos que la sociedad impone a hombres y mujeres ni semejantes sus efectos; de modo que cuando examinamos el efecto de una dolencia determinada o de un tratamiento específico debemos tomar en cuenta el escenario social en lo que a género respecta. En el campo de la salud este es un factor de complejidad.

Es esencial tener en cuenta que la situación de salud de cada mujer es diferente y que no se debe usar solamente la edad para su clasificación universal, diagnóstico o tratamiento de enfermedades. Aún más importante es que no se use la edad para justificar la discriminación o el trato inequitativo. 
Las principales causas de morbilidad y mortalidad de las mujeres de mayor edad en los países latinoamericanos son parecidas a la de países desarrollados: enfermedad cerebrovascular, enfermedades crónicas discapacitantes, enfermedades mentales, entre otras. Lamentablemente, los programas de salud para mujeres de países en vías de desarrollo están más orientados a aquellas en edad fértil. Algunas veces sólo los adultos mayores masculinos pueden utilizar o tienen derecho a los servicios de salud, o bien, sólo se tratan algunas patologías más frecuentes en mujeres ancianas.

La salud en la vejez es un proceso acumulativo. La perspectiva del ciclo de vida reconoce que la salud de las mujeres de mayor edad dependerá en gran medida de su estilo de vida y comportamiento. El cuidado de la salud en las mujeres más jóvenes es crucial en cualquier programa que otorgue prioridad a la prevención de la enfermedad en lugar de a su tratamiento, situación que no ocurre en nuestros países latinoamericanos.

\section{Papel de la bioética}

Para la atención de salud de las adultas mayores se deben considerar los principios de beneficencia y justicia. Estos contribuirán a la atención individual y a la formación de sistemas sanitarios (o políticas sanitarias) de carácter equitativo, de tal modo que se distribuyan los recursos de acuerdo con las necesidades de:

- Población vieja.

- Mayoritariamente mujeres.

- Sin políticas de salud (en la mayoría de los casos).

No se es justo y no se hace el bien si no hay eficiencia. Para alcanzarla se deben considerar los factores propios de las adultas mayores:
Factores de salud:

- Estilo de vida: Dieta y nutrición, peso, nivel de actividad física y de estrés físico y emocional. Hábitos tóxicos (consumo de tabaco, alcohol o drogas) y actividad sexual.

- Trabajo: En la mayoría de las sociedades latinoamericanas el trabajo remunerado de las mujeres concluye alrededor de los 60 ó 65 años; sin embargo, la mayoría trabaja hasta que muere, hecho no reconocido por el sector salud y/o las estadísticas laborales. A esta edad son mayormente responsables de las tareas domésticas en el hogar y, en algunas ocasiones, se encargan del esposo en igual o peores condiciones que ellas (y de mayor edad).

- Protección social: Las mujeres tienen menos acceso a ésta, lo que repercute negativa y seriamente en su salud durante la vejez. Ello se debe a que, en los países en desarrollo, su trabajo está concentrado en los sectores informal, agropecuario y de servicios.

- Responsabilidades de atención a la familia: Es parte del trabajo invisible (doméstico) que realizan las mujeres (el cuidado de la pareja enferma, de los niños y de los nietos) situación que puede tener consecuencias graves en su salud física y psicológica.

\section{Estado de vida:}

- Estado socioeconómico: Incluye desde el nivel de ingreso durante su vida hasta el lugar que ocupa en la comunidad, sin olvidar el grupo étnico a que pertenece (el valor atribuido al género y a la raza repercute claramente en la salud y calidad de vida). Es un factor determinante de la salud, particularmente entre las mujeres de mayor edad, que, una vez jubiladas, dependen de la seguridad social, de sus ahorros o de otros 
miembros de su familia (quienes pueden ser pobres también).

- Estado civil: Ser solteras (viudas, divorciadas, abandonadas o nunca casadas) es una realidad para muchas mujeres de mayor edad. Sus efectos sobre la salud son difíciles de cuantificar, pero pueden incluir la falta de recursos, depresión, falta de movilidad y pobreza.

- Acceso a los servicios de salud: La salud de la mujer durante la vejez dependerá de este acceso durante toda su vida. Esto incluye no solamente los recursos para pagarlo, sino también la forma de trato que reciben por parte de los profesionales de la salud. Hay una tendencia a prescribir medicamentos para las mujeres de mayor edad, en lugar de buscar las causas de sus problemas. En vez de hacer la vida más agradable, los efectos secundarios y los peligros de combinar diferentes medicamentos pueden tener repercusiones perjudiciales sobre su calidad de vida.

\section{Más factores}

- Historia clínica y familiar: Debido a que constituye un factor de riesgo importante para ciertas enfermedades (cáncer de mama, por ejemplo), es esencial contar con la historia de la paciente, tomando en cuenta: enfermedades y lesiones de la infancia, uso de diferentes medicamentos, salud reproductiva, exposición a la violencia, intervenciones quirúrgicas, entre otros aspectos. Todos estos eventos tienen un impacto en su salud a medida que envejece y deben tomarse en cuenta al evaluar su perfil de salud.
- Salud mental: La soledad o la dependencia de otros miembros de la familia también afecta la salud mental de las mujeres mayores. Hay que tomar en cuenta siempre los efectos acumulativos, ya que, desde su juventud, han estado expuestas a la violencia de género, al trabajo no remunerado y a la discriminación.

Es necesario modificar para todas las mujeres jóvenes la situación de abandono y discriminación, independientemente de su posición social, su etnia o su lugar de residencia. Debemos aceptar el reto y sumar esfuerzos en la construcción de un nuevo contexto: una sociedad justa, solidaria, contenedora y equitativa, con su centro en la condición humana. De manera que sea factible que ellas envejezcan con la máxima calidad de vida y con un mínimo de enfermedad, sin que ello signifique hacer insostenibles los costos de salud, como es la tendencia actual.

La salud en el envejecimiento requiere de la perspectiva de género para una mejor comprensión. Con esta visión reconocemos dificultades y desigualdades posibles de solucionar poniendo en práctica los principios bioéticos.

\section{Agradecimientos}

Trabajo realizado durante el Programa Internacional de Formación en Ética de la Investigación Biomédica y Psicosocial del Centro Interdisciplinario de Estudios en Bioética de la Universidad de Chile, financiado por el Program Training Grant 1R25TW06056-01, Fogarty International Center (FYC) de los Institutos Nacionales de Salud (NIH) de Estados Unidos. 


\section{Bibliografía}

Bonita R. Women, Aging and Health: Achieving Health across the Lifespan. Ginebra: Organización Mundial de la Salud; 1998.

Bronfman E. Envejecimiento y género: algunas reflexiones. Revista Mujer Salud RSMLAC 2005; 2(3): 66-70.

González A, Rodríguez L. Derribar los mitos en la tercera edad. Un reto para la ética médica. GEROINFO 2006: 2(2).

Lolas F. Las dimensiones bioética de la vejez. Acta Bioethica 2001; 7(1): 57-69.

Lolas F. Escritos sobre vejez, envejecimiento y muerte. Iquique: Ediciones Campus; 2002.

Lolas F. El desafío bioético de la equidad: su relevancia en la salud pública. En: Salud pública: enfoque bioético. Caracas: Editorial Disinlimed CA; 2005: 35-41.

Lolas F. Bioética: el diálogo moral en las ciencias de la vida. Santiago de Chile: Mediterráneo; 2003.

Málaga H. Intervenciones para combatir la exclusión individual y su impacto en la exclusión social. En: Salud pública: enfoque bioético. Caracas: Editorial Disinlimed CA; 2005: 41-50.

Matamala MI. Mujer, menopausia y una larga vida. Necesidad de una nueva ética occidental. Revista Mujer Salud RSMLAC 2005; 2(3): 60-65.

Recibido el 18 de mayo de 2006.

Aceptado el 22 de junio de 2006. 